\title{
Viral proteomics: The emerging cutting-edge of virus research
}

\author{
ZHOU ShengTao ${ }^{1,2 \dagger}$, LIU Rui $^{2 \dagger}$, ZHAO Xia $^{1 *}$, HUANG CanHua $^{2 *} \&$ WEI YuQuan ${ }^{2}$ \\ ${ }^{1}$ Department of Gynecology and Obstetrics, West China Second Hospital, Sichuan University, Chengdu 610041, China; \\ ${ }^{2}$ State Key Laboratory of Biotherapy, West China Hospital, Sichuan University, Chengdu 610041, China
}

Received June 13, 2010; accepted December 3, 2010

\begin{abstract}
Viruses replicate and proliferate in host cells while continuously adjusting to and modulating the host environment. They encode a wide spectrum of multifunctional proteins, which interplay with and modify proteins in host cells. Viral genomes were chronologically the first to be sequenced. However, the corresponding viral proteomes, the alterations of host proteomes upon viral infection, and the dynamic nature of proteins, such as post-translational modifications, enzymatic cleavage, and activation or destruction by proteolysis, remain largely unknown. Emerging high-throughput techniques, in particular quantitative or semi-quantitative mass spectrometry-based proteomics analysis of viral and cellular proteomes, have been applied to define viruses and their interactions with their hosts. Here, we review the major areas of viral proteomics, including virion proteomics, structural proteomics, viral protein interactomics, and changes to the host cell proteome upon viral infection.
\end{abstract}

virus, proteomics, virion proteomics, virus host interaction

Citation: Zhou S T, Liu R, Zhao X, et al. Viral proteomics: The emerging cutting-edge of virus research. Sci China Life Sci, 2011, 54: 502-512, doi: 10.1007/ s11427-011-4177-7

Viruses are not only notorious for their pathological nature and associated diseases, but also for their role as model systems for studying molecular events and identifying critical cellular machineries. Previous investigations have provided insights into the structure, function, and protein interactions of individual viral proteins. More significantly, these studies have partially elucidated the associated positive and negative gene regulation, chaperone activity, repressor-operator interactions, DNA replication, transcriptional elongation and termination, RNA splicing, immune signaling, and oncogenic transformation [1]. However, only a limited number of viral proteins have been extensively studied to date. A large body of information on viruses and virus-host interactions remains untapped. The analysis of such data will increase our understanding of fundamental biological processes.

Proteomics is an emerging high-throughput technology

$\dagger$ Contributed equally to this work

*Corresponding author (email: xia-zhao@126.com; hcanhua@ hotmail.com) that complements genomics exploration to evaluate protein-protein interactions and cellular alterations on a global scale, allowing the discovery of previously unannotated connections. Progress in mass spectrometry-based proteomics strategies not only revolutionized the investigation of specific proteins, but also enabled global protein expression analysis [2,3]. However, proteome research is still somewhat held back by the intrinsically complicated nature of proteins, the heterogeneity in subcellular location of proteins, and the variable sensitivity in identifying less abundant proteins in the presence of highly abundant proteins [4,5]. Recent advances in comparative proteomics and quantitative proteomics have addressed some of these drawbacks and have provided seminal weapons for protein investigation.

The application of proteomics strategies in viral research has, for some time, attracted increasing attention worldwide. Proteomic studies of the host upon viral infection have delineated the molecular interactions between the virus and the host, thus providing hints for the detection, diagnosis, 
and therapeutics of infectious diseases. In this review, we have divided viral proteomics into virion proteomics and host-virus interactions. We have also revisited some of the pros and cons of the available proteomics methods in virus investigations.

\section{Virion proteomics}

Viruses evolved to adapt to harsh host environments by packaging their genetic material with positively charged capsid proteins and, under some circumstances, an additional tegument layer and extra membrane coats. While capsids consist of protein subunits encoded by viral genomes, envelopes comprise not only viral proteins, but also host cell proteins, lipids, and carbohydrates. Viruses orchestrate these molecular compositions to complete a wide spectrum of functions during infection, replication, and propagation, including assembly, exit, transmission, attachment, penetration, and uncoating [6]. Thus, viral protein composition analysis is useful for elucidating virus structure and for determining modification of virus protein expression during attachment and entry of the virus into host cells.

Compared with eukaryotic cell proteomes, virus proteomes are simple and highly conserved. However, relatively few virion proteomes have been characterized to date. Conventionally, determining the global protein composition of a specific virus particle involves pure virus preparation, detergent denaturation, electrophoretic separation, and identification of protein bands by immunoblotting or by se- quencing with Edman degradation [7]. In recent years, new approaches that utilize a combination of techniques have provided potent tools for virion research. Two-dimensional electrophoresis (2DE) coupled with identification of protein spots using mass spectrometry, also termed "the shotgun approach", has generated robust amounts of virus protein information. Another novel viral proteomics strategy is based on the analysis of differential enzymatic digestions of whole virions, or solubilized and deconvoluted virion proteins. The protein extracts thus obtained are fragmented into peptides that are further separated by 1D or multidimensional liquid chromatography (LC), and analyzed by MS. Both technologies require subsequent database search algorithms to define peptide fragmentation patterns and the corresponding proteins, and require specialized bioinformatics analysis. These techniques have been confirmed to be useful for the exploration of virion proteomes (Table 1).

\section{$1.1 \quad$ Non-enveloped viruses}

To date, a few proteomic studies have attempted to analyze virions from non-enveloped eukaryotic viruses, the most studied of which is adenovirus. In 2002, one group employed LC-MS/MS with both one-dimensional and twodimensional chromatographic separations to characterize the protein complement of adenovirus type 5 virions. Eleven protein species were identified from 154 peptides, and all of the major viral proteins were found; however, the $23-\mathrm{kD}$ viral protease was detected only after $2 \mathrm{D}$ separation. The researchers concluded that LC-MS/MS was a powerful

Table 1 Virion proteomes identified by MS-based proteomics strategies

\begin{tabular}{|c|c|c|c|c|c|}
\hline Virus & Host & $\begin{array}{c}\text { Protein number iden- } \\
\text { tified by proteomics } \\
\text { (novel proteins) }\end{array}$ & Host proteins detected & Separation technique/mass spectrometry & Ref. \\
\hline Adenovirus type 5 & Human & $11(2)$ & $\mathrm{ND}^{\mathrm{a})}$ & RPLC/LC-ESI-MS/MS & [8] \\
\hline \multirow[t]{3}{*}{ Vaccinia virus } & Human & $80(13)$ & 24 & 2D-LC (SCX and RP); ESI-MS/MS & [10] \\
\hline & Human & $75(10)$ & 23 & 2D-LC (SCX and RP)/ESI-QTOF-MS & [11] \\
\hline & Human & $63(2)$ & ND & $\begin{array}{l}\text { 1DE or RPLC/MALDI-TOF/TOF-MS, } \\
\text { LC-ESI-QTOF-MS or LC-ESI-QIT-MS }\end{array}$ & [12] \\
\hline Human cytomegalovirus & Human & $21(6)$ & ND & $\begin{array}{c}\text { Negative viscosity-positive density gradient cen- } \\
\text { trifugation; 1D-GE/ MS }\end{array}$ & [13] \\
\hline Murine cytomegalovirus & Mouse & $58(20)$ & 7 & 1DE/nano-RP-HPLC-ESI-QTOF-MS & {$[14]$} \\
\hline EBV & Human & 41 (ND) & 6 & ICAT/MS/MS & [15] \\
\hline $\begin{array}{c}\text { Kaposi's sarcoma associated } \\
\text { herpes virus }\end{array}$ & Human & $24(5)$ & 21 & 1DE/nano-RP coupled to ESI-MS/MS & [16] \\
\hline Rhesus radiovirus & Monkey & $33(3)$ & ND & 2D-LC (SCX and RP)/ESI-MS/MS & {$[17]$} \\
\hline White spot syndrome virus & Shrimp & $45(13)$ & ND & RPLC/MALDI-TOF/TOF-MS & [19] \\
\hline SARS coronavirus & Human & $4(4)$ & ND & 2D-GE/LC-ESI-MS & {$[20]$} \\
\hline \multirow[t]{2}{*}{ HIV-1 } & Human & $14(\mathrm{ND})$ & 253 & 1DE/MALDI-TOF RPLC-ESI-MS/MS & {$[21]$} \\
\hline & Human & $10(\mathrm{ND})$ & 12 & RPLC-MS/MS & {$[22]$} \\
\hline Murine gammaherpesvirus 68 & Mouse & $17(5)$ & 5 & 1D-GE/LC-MS/MS & [23] \\
\hline Human cytomegalovirus & Human & $71(12)$ & 70 & 2D-LC(SCE and RP)/FTICR/MR and LC-MS/MS & {$[24]$} \\
\hline
\end{tabular}

a) ND, not determined. 
tool for identifying whole virion proteins that are present at copy numbers of 10 or more [8].

\subsection{Enveloped DNA viruses}

Chronologically, the first proteome of a DNA virus particle to be sequenced was an ictalurid fish herpes virus, the channel catfish virus, in 1995. Purified virions were solubilized with NP-40, separated by gel electrophoresis, electroblotted, and the bands digested with different proteases including trypsin, Lys-C, Arg-C, or glu-C to generate a broad spectrum of peptide fragments. The fragments were subsequently analyzed using MALDI-MS. The peptide mass fingerprint was used to search the Swiss-Prot database, and a total of 16 primary structural proteins, including mature capsid proteins, protein kinases, $\mathrm{C} 3 \mathrm{HC} 4$ zinc-binding protein, and actin were identified [9]. More importantly, the above-mentioned combined strategy of MS and sequence database searching has now become widely applied for the analysis of in the proteomes of such DNA virions as poxviruses and herpes viruses.

With regard to poxviruses, although the complete genomic sequences of most poxviruses have been available for some time, only a limited number of studies have attempted to comprehensively profile the protein composition of poxvirus virions [25]. Early proteomics studies attempted to analyze the protein composition of purified vaccinia virus (VV). However, they were unable to perform a complete structural analysis of all the protein components of the virions, probably because the limited availability of advanced purification methodologies and the limitations of the technologies available for protein identification at the time. Recently, three different groups with different approaches, instruments, and digestion protocols reported the comprehensive structural analysis of the protein composition of the infectious mature virion of VV [10-12]. For instance, Resch et al. [10] detected 80 vaccinia virus-encoded proteins, representing $37 \%$ of the 218 genes annotated in the complete genome sequence, using denaturation with SDS or urea, fractionation with solid-phase extraction columns, and analysis by LC-ESI-LTQ Fourier transform MS. Among them were 13 proteins were not previously described as being part of the virion. H5, F8, E3, E6, and A6 were not previously reported as being virion-associated proteins, while N1, C22, A11, A19, M1, A50, E9, and F4 had been described as absent or unlikely to be associated with the virion.

The growing number of studies investigating herpes viruses has also resulted in a more comprehensive understanding of the structure of the herpes virion, as well as the function of each viral protein [26]. Herpes viruses investigated by proteomics strategies include human cytomegalovirus (CMV) (HCMV) [13] and murine cytomegalovirus of the beta herpesviruses [14], Epstein-Barr (EB) virus (EBV) [17], Kaposi's sarcoma-associated herpesvirus
(KSHV) [16,27,28], rhesus monkey rhadinovirus (RRV) [17], and murine gamma herpesvirus 68 (MHV68) of the gamma herpesviruses [29]. However, no virion proteomics studies of alpha herpesviruses have yet been documented, including the intensively studied herpes simplex virus type 1 (HSV-1).

Other enveloped DNA viruses have been studied using an integrated proteomics strategy, for example, white spot syndrome virus (WSSV). WSSV is a double-stranded DNA virus that contains a $305-\mathrm{kb}$ circular DNA, which putatively encodes 181 ORFs. In 2002, Huang et al. [18] separated the purified WSSV virions by SDS-PAGE, excised 24 protein bands, and subjected them to in-gel trypsin digestion, MALDI-TOF, and electrospray ionization tandem mass spectrometry. Eighteen proteins corresponding to ORFs of the WSSV genome were identified, among which were 14 proteins of unknown functions. Temporal analysis implied that all the genes were transcribed in the late stage of WSSV infection, except for vp121. Of the 14 newly-identified proteins, VP466 was further characterized as a novel envelope protein. More recent research described the application of the isobaric tags for relative and absolute quantification (iTRAQ ${ }^{\mathrm{TM}}$ ) approach to define the location of proteins in WSSV [19]. Li et al. [19] reported 45 viral proteins, including 13 novel proteins associated with the virion. In particular, they identified 23 envelope proteins and six nucleocapsid proteins, including 12 novel envelopes and two novel nucleocapsid proteins. This study exemplifies the successful application of quantitation techniques to investigate the localization of virion proteins.

\subsection{Enveloped RNA viruses}

Unlike the wide spectrum of proteomics research of virions of DNA viruses, only a limited number of proteomic studies have been carried out on RNA viruses, primarily because of their simpler structures and the fewer numbers of proteins in RNA virions. Therefore, high-throughput proteomics approaches to investigate RNA virion proteomes have seemed unnecessary. Currently, two types of enveloped RNA viruses have been intensively analyzed: severe acute respiratory syndrome-associated corona virus (SARS-CoV) and HIV-1.

In 2004, Zeng et al. [20] utilized one-dimensional electrophoresis followed by ESI MS/MS identification or a shotgun strategy with two-dimensional liquid chromatography-ESI MS/MS to identify the structural proteins of SARS coronavirus (SARS-CoV) isolated from Vero E6 cells infected with the BJ-01 strain of the virus. All four of the predicted structural proteins (nucleocapsid $(\mathrm{N})$, spike $(\mathrm{S})$, membrane (M), and envelope (E)) were identified, and a novel phosphorylated site of the $\mathrm{M}$ protein was revealed. This combination of gel-based and non-gel approaches provides a fast and complimentary tool, which can be expected to be widely applied in the analysis of other viruses. An- 
other comprehensive proteomic analysis on the structural proteins of SARS-CoV uncovered three of the structural proteins ( $\mathrm{S}, \mathrm{N}$, and $\mathrm{M}$ ). Four glycosylation sites of the $\mathrm{S}$ protein were discovered by comparing the mass spectra before and after deglycosylation of the peptides with PNGase F digestion. Surprisingly, a number of shorter isoforms of the $\mathrm{N}$ protein were noted by SDS-PAGE and identified by mass spectrometry characterization [30]. This study suggested that the identification of virus-specific proteins by MS could be effectively employed as a diagnostic method for rapid screening of highly infectious viruses, virus-infected cells, or even biofluids containing viruses.

HIV jeopardizes the host immune system by specifically attacking macrophages and $\mathrm{CD}^{+} \mathrm{T}$ cells, and multiple groups have defined a robust number of host cell proteins in HIV virions. One group used LC MS/MS on whole virions to gain an overview of the HIV-1 proteome. All known viral and host-cellular proteins, as well as novel components of HIV-1, were identified and further confirmed by traditional biochemical methods [21]. In another study, highly purified HIV-1 virions produced from human monocyte-derived macrophages (MDM) were analyzed [22]. Liquid chromatography-linked tandem mass spectrometry identified a plethora of cellular proteins, including 33 previously annotated proteins in HIV-1 preparations from other cell types. These proteins were involved in such cellular structures and functions as the cytoskeleton, adhesion, signaling, intracellular trafficking, chaperoning, metabolism, ubiquitin/proteasome, and immune response systems. Other identified proteins included annexins, annexin-binding proteins, Rab proteins, and proteins involved in membrane organization, vesicular trafficking, and late endosomal function, Apolipoprotein E, which participates in cholesterol transport, immunoregulation, and modulation of cell growth and differentiation, was also identified. These results provide significant leads for follow-up experiments on HIV-1 assembly, infection, and pathogenesis.

\subsection{Bacteriophages}

Bacteriophages (phages) are the most abundant and diverse form of life and have indispensable roles in the microbial world. A series of studies have examined the structural proteomes of bacteriophages. Savalia et al. [31] utilized liquid chromatography and electrospray ionization mass spectrometry to analyze the virion proteins of phiEco32, a novel Escherichia coli bacteriophage. Two phiEco32-encoded proteins that interact with the host RNA polymerase were defined. One is an ECF family $\sigma$ factor that might be responsible for the transcription of certain viral genes, and the other is a novel RNA polymerase-binding protein and transcription. Roberts et al. [32] explored the proteome of coliphage T1 using isoelectric focusing to separate the components of the virion, followed by MALDI-TOF. T1 was expected to consist of 13-15 structural proteins in the mature virion. Correspondingly, the 2DE analysis of the T1 virion performed in the same study resolved 15 proteins. Subsequent mass spectrometry revealed four abundantly expressed proteins: the major tail protein (gp41), a major capsid component (gp49), the major head subunit (gp47), and a protein associated with the major head subunit (gp48) [10]. In 2006, Naryshkina et al. [33] determined the sequence of the 152372 bp genome of pYS40, a lytic tailed bacteriophage of Thermus thermophilus. pYS40 contains 170 putative ORFs and three tRNA genes. Functions for a quarter of the pYS40 gene products were putatively attributed, based on similarity to proteins of known function from diverse phages and bacteria. A cluster of proteins was identified as being involved in nucleotide salvage, such as flavin-dependent thymidylate synthase, thymidylate kinase, ribonucleotide reductase, and deoxycytidylate deaminase. Others were involved in DNA replication, such as DNA primase, helicase, type A DNA polymerase. The increasing number of such studies will provide invaluable knowledge complementing the annotation of phage genomes and allowing for comparative genomics studies.

Collectively, although a growing number of eukaryotic viruses and phages have been sequenced, only a small proportion of them have been characterized at the protein level. The identification of previously unknown gene products is important for our understanding of virion structures, and more importantly, is pivotal to the investigation of virus and host interaction. In addition, the development of virion proteomics might also provide potent tools for diagnosis, and could generate novel insights into the therapeutics of virus-induced infectious diseases.

\section{Host-virus interactions}

Host-virus interactions have been explored ever since the discovery of viruses in 1898, when Martinus Beijerinck described tobacco mosaic virus [34]. However, only after the invention of the electron microscope, protein biochemistry, and ultimately, nucleic acid sequencing, has the systematic molecular definition of these interactions become achievable. Many studies have focused on the interaction of viral proteins with viral and host cellular proteins (interactomics) and on alterations in cellular protein abundance during infection. Despite of the documentation of many thousands of protein-protein interactions between virus and human proteins, we are still far from a complete comprehension of the different kinds of virus biology. Thus, novel technology suitable for molecular biology is vital for uncovering the complexities of each infection process. The most frequently used techniques for interactomics include yeast two-hybrid (Y2H) screening and co-immunoprecipitation of interacting proteins, followed by MS analysis. In particular, the $\mathrm{Y} 2 \mathrm{H}$ method can be applied to highthroughput screening of interactions between any two pro- 
teins. As for host cell proteomics, 2DE coupled with MS and stable isotope labeling methods have been successfully applied (Table 2).

\subsection{Interactomics}

The most widely applied methods to study protein-protein interactions on a proteome-wide scale include $\mathrm{Y} 2 \mathrm{H}$ screening and tagged protein immunoprecipitation, coupled with protein identification with $\mathrm{MS}$. While the $\mathrm{Y} 2 \mathrm{H}$ method fa- cilitates the mapping of binary or pair-wise interactions, affinity capturing approaches, followed by MS-based protein identification methodologies, enable the investigation of protein interaction complexes comprising more than two molecules (Figure 1).

\subsection{Yeast two-hybrid screening}

The Y2H system was first introduced by Fields and Song [35] in 1989, and delineates binary physical interactions between

Table 2 Pros and cons of distinct proteomics strategies for virus research

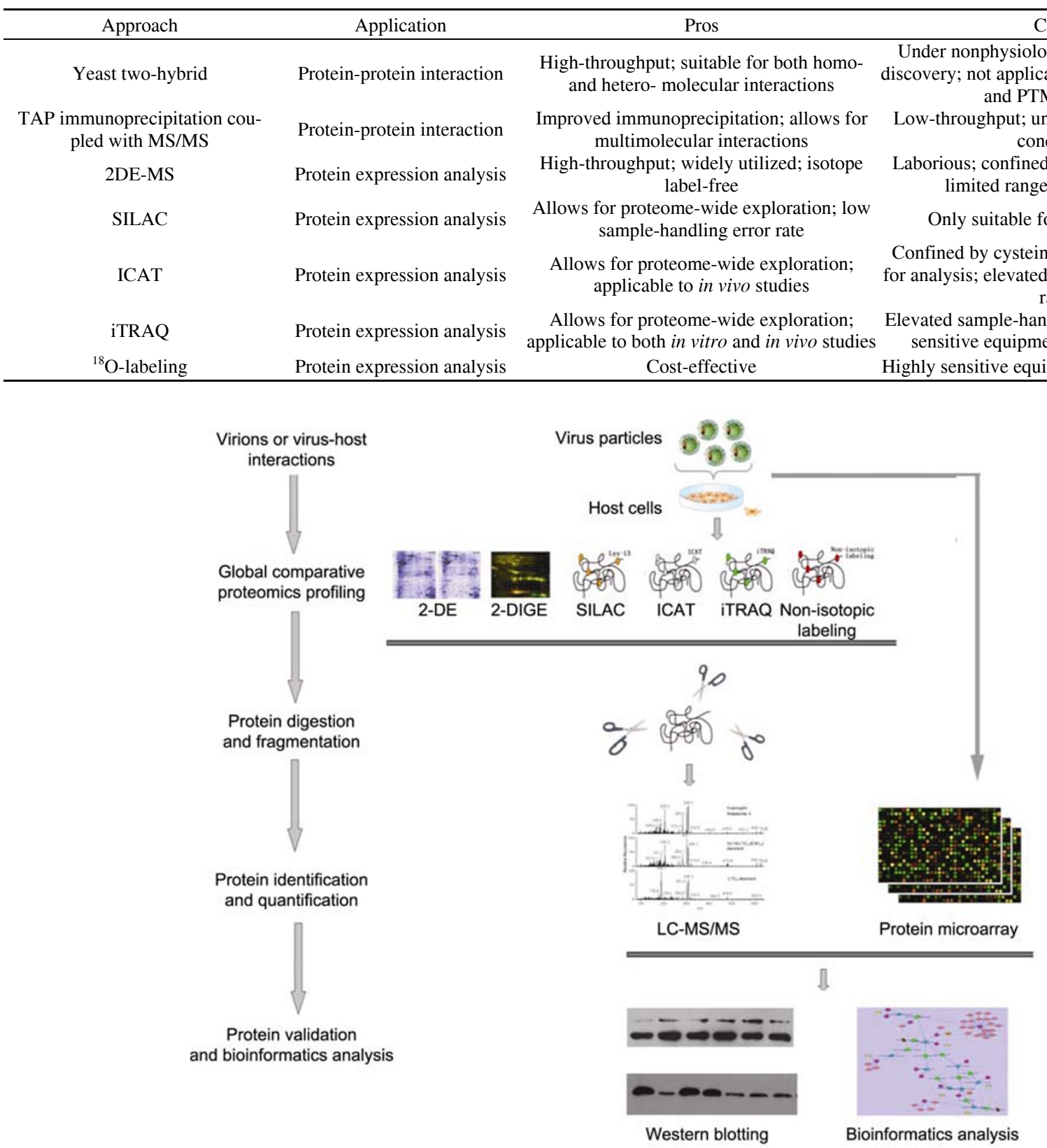

Figure 1 The workflow of proteomics strategy to analyze global protein expression. The protein expression profiles of virions or virus-host interaction samples are analyzed by global proteomics strategies (e.g., 2-DE-MS, SILAC, ICAT, iTRAQ, or protein array). The expression patterns of altered proteins are then substantiated through such tools as Western blotting and can be systematically analyzed by bioinformatics software. 
proteins. The major advantages of the $\mathrm{Y} 2 \mathrm{H}$ system are the facts that it allows one bait to be matched against multiple preys, and that both homo- and heterodimeric interactions can be detected. Moreover, the screens are largely independent of the optimal experimental conditions compared with affinity purification research, because both bait and prey libraries are expressed at comparable levels. Although this may generate nonphysiological protein levels, the increased expression levels might also enhance the sensitivity of the system [36]. Despite its strengths, the Y2H system is defective in its capacity for detecting interactions. On the one hand, it cannot detect cooperative interactions between more than two proteins because of the binary nature of the technique. On the other hand, the interaction occurs in the nucleus, which poses problems for the expression of integral membrane proteins. In addition, certain proteins and transcription factors might independently enable the transcription of the reporter. In addition, ectopically expressed proteins sometimes evade post-translational modification (PTM), thereby ruling out dynamic, PTM-dependent, binary interactions [37].

The Y2H system has been widely applied in the study of global proteome interaction of various viruses. The most recent virus interaction screening database includes $\mathrm{T} 7 \mathrm{bac}-$ teriophage, VV, and KSHV \& varicella-zoster virus. As early as 1996, Bartel et al. [38] used the yeast two-hybrid system on a genome-wide scale to identify 25 interactions among the proteins of Escherichia coli and bacteriophage T7. Among these, a set of six interactions connected proteins that function in DNA replication and DNA packaging. Several of the interactions reflect intramolecular associations of different domains of the same polypeptide, suggesting an important role of the two-hybrid assay in the analysis of protein folding. McCraith et al. [39] performed a comprehensive two-hybrid analysis to detect interactions among proteins of vaccinia virus. They constructed an array of yeast transformants that comprised each of the 266 putative viral ORFs as Gal4 activation domain hybrid proteins. The array was individually mated to transformants containing each ORF as a Gal4-DNA-binding domain hybrid. Diploids expressing the two-hybrid reporter genes were defined. Of the 70000 combinations, 37 protein-protein interactions were found, including 28 that were previously unannotated. The interacting proteins could be functionally categorized into DNA replication, transcription, virion structure and morphogenesis, and virus-host interactions. Another study investigated intraviral protein interactions in herpes viral proteins, KSHV, and varicella-zoster virus (VZV). Eightynine ORFs from the KHSV genome were tested for 12000 viral protein interactions, leading to the detection of 123 nonredundant interacting protein pairs. Of the 69 ORFs in VZV and 10000 tested bait-prey pairs, 173 nonredundant intraviral protein interactions were found. A database of all predicted viral intraprotein interactions and previously established viral protein-human cellular protein interactions has been set up. This database facilitated the prediction of 20 interactions between eight KSHV and 20 human proteins [28]. These studies illustrate that the global yeast two-hybrid screening of protein-protein interactions can be applied to the analysis of emerging, more complex, genomes.

\subsection{Tandem affinity purification}

In 2004, Dziembowski et al. [40] documented the tandem affinity purification (TAP) procedure, which employs a fusion tag consisting of two sites separated by a protease cleavable spacer. This technology has been applied in a variety of virus research. Jorba et al. [41] purified the intracellular complexes formed by the influenza polymerase in human cells by the TAP approach. The purified complexes contained the heterotrimeric polymerase and a series of associated proteins that were not apparent in purifications of control untagged polymerase. Further identification of influenza polymerase-associated proteins was achieved by MALDI-MS and validated by Western blotting. The roles of these proteins in influenza infection were substantiated by colocalization with virus ribonucleoproteins in infected human cells. The majority of the associated human factors were nuclear proteins involved in cellular RNA synthesis, modification, and nucleocytoplasmic export, except for cytosolic proteins involved in translation and transport. The interactions annotated in this proteomic research suggest a significant role for the influenza polymerase in the infection cycle other than in RNA replication and transcription. In research by Mayer et al. [42], strep-tagged viral nucleoprotein (NP-Strep) bait and TAP-tagged polymerase subunit bait (TAP tag) were applied to explore the interacting proteins of influenza A viral ribonucleoprotein (vRNP). Forty-one proteins were identified using NP-Strep bait, and four proteins were found using TAP-tagged bait. These included importin- $\beta 3$ and PARP-1, the two polymerase-associated factors. These studies proved the effectiveness of TAP purification in protein interaction analysis. However, the application of this technology is limited because of its relatively low throughput compared with other methods.

\subsection{In vitro pull-down assay}

While the $\mathrm{Y} 2 \mathrm{H}$ method serves as the initial identification of protein-protein interactions, further confirmation of the interaction requires other pull-down assays. These methods involve the expression of one or both of the proteins in another organism, usually $E$. coli, and the addition of affinity tags such as His6 and GST to the N- or C-terminus of the expressed proteins to improve purification and identification. If only one of the proteins is expressed recombinantly, a native source of the second partner must be available [37]. Several studies have used this method to investigate protein-protein interactions. A recent report unraveled the expression of pituitary tumor-transforming gene 1 (PTTG1)/ 
securin in hepatitis B virus (HBV)-associated liver diseases using glutathione S-transferase pull-down and co-immunoprecipitation experiments [43]. They concluded that the interaction between PTTG1 and the Skp1-Cul1-F-box ubiquitin ligase complex (SCF) was partially disrupted, possibly through a mechanism involving protein-protein interactions of HBx with PTTG1 and/or SCF. Thus, pull-down experiments can validate $\mathrm{Y} 2 \mathrm{H}$ interaction results in virus research. This strategy is potentially useful for the definition of binding partners that conduct signals, as well as for interactions that occur when the host and the virus fuse [26].

\subsection{Dynamic viral protein interaction through GFP tags}

Although the $\mathrm{Y} 2 \mathrm{H}$ system provides a high-throughput tool for protein-protein interaction screen, it cannot monitor the dynamic interactions that occur upon virus infection. One group constructed a modified immunoprecipitation protocol to unravel the dynamic viral protein interaction using a green fluorescent protein (GFP)-tagged virus [44]. This protocol involved tagging the virus with GFP-encoded proteins, visualizing the proteins' locations in the host cell at distinct infection stages, isolating interacting proteins using GFP affinity columns, and protein validation by MS. This study examined the interaction of the nsP3 protein of $\alpha$ virus, Sindbis, with mammalian proteins at different times during infection. They demonstrated that a host factor was recruited to nsP3-containing complexes in a time-dependent fashion. This process was concomitant with a specific early and continuous recruitment of G3BP, a nuclear transport factor, and a subsequent recruitment of 14-3-3 proteins. These conclusions not only confirmed that Sindbis virus infection can modify RNA transport, but more significantly, revealed the utility of novel high-throughput methodologies to verify protein-protein interactions in host cells infected by viruses.

\subsection{Protein interaction screens \& functional screens: RNAi}

The previously described technologies only offer a descriptive view of protein-protein interactions. They cannot characterize the physiological significance of these interactions, which are often elucidated by mutations or other functional experiments. In recent years, the development of RNAibased methods for protein knockdown in mammalian cells has allowed unparalleled flexibility in the study of protein function. Large siRNA libraries are now available that allow the knockdown of all proteins known to be encoded by the human genome. These libraries have been used to provide mechanistic explanations by depleting RNAs of defined genes. Together with bioinformatics analysis, this approach has played a pivotal role in identifying host factors required for such viruses as HIV, flaviviruses, and the H1N1 virus. Thus, RNAi screens and protein interaction screens perfectly complement protein-protein interaction screens [45]. For example, Brass et al. [46] and Konig et al. [47] conducted two independent investigations that systematically screened for human genes indispensable for HIV infection and replication. They identified 284 and 295 required cell factors, respectively, with an overlap of 13 genes. Integration of RNAi data and protein interaction data generated a host-pathogen interaction network comprising 213 functionally validated and 169 predicted HIV host cellular nodes. These nodes were cross-linked via 2291 binary protein interactions and 318 interactions to HIV-encoded proteins. This RNAi analysis revealed the biological context through phenotypes provided simultaneous mechanistic explanations for these phenotypes.

\section{Protein-expression analysis}

The combination of LC-MS and sequence database searching has been successfully applied to the analysis of global protein expression. The major methods used for protein expression analysis include 2DE-based separation of proteins and stable isotope labeling techniques (Figure 2).

\subsection{DE-MS}

Currently, protein isolation by 2DE coupled with MS-based analysis of differentially expressed spots, is the most widely utilized among a wide spectrum of protein-expression analysis technologies, primarily because of its simplicity and wide applicability. Viruses studied by this approach comprise $\mathrm{HBV}$, EBV, SARS-CoV, HCV, and HIV-1. In 2008, Tong et al. [48] successfully utilized 2-DE and MS/MS analysis to compare and identify differentially expressed proteins between an HBV-producing cell line, HepG2.2.15, and its parental cell line, HepG2, to determine the effects of HBV replication on host cell-protein expression. Sixty-two spots were positively identified by MS/MS analysis. Cluster and metabolic/signaling pathway analysis divided the proteins into three groups: retinol metabolism pathway, calcium ion-binding proteins, and proteins associated with protein degradation pathways. This study provided novel insights into the mechanisms underlying $\mathrm{HBV}$-host cell interactions and the development of HCC [11]. In another study, 2D-GE-MALDI-TOF-MS was applied to clinical HBV-associated hepatocellular carcinoma. One hundred protein spots, corresponding to 80 distinct gene products, were identified. Among these 100 proteins, proliferating cell antigen and stathmin 1 were further corroborated by Western blotting. In an attempt to profile EBV-induced cellular proteome alterations, EBV transformed B-lymphoblastoid cell lines (LCLs) were used as a model system to identify the proteins essential for the im- 
Yeast two-hybrid method

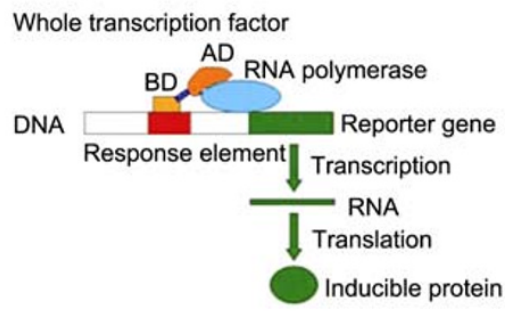

Separate bait-BD and trap-AD

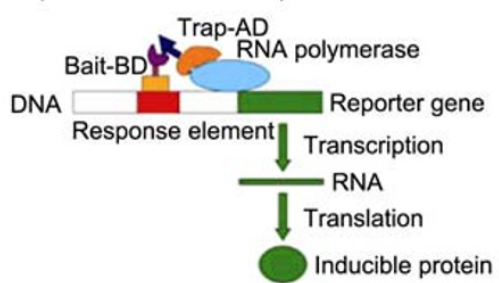

TAP tag purification

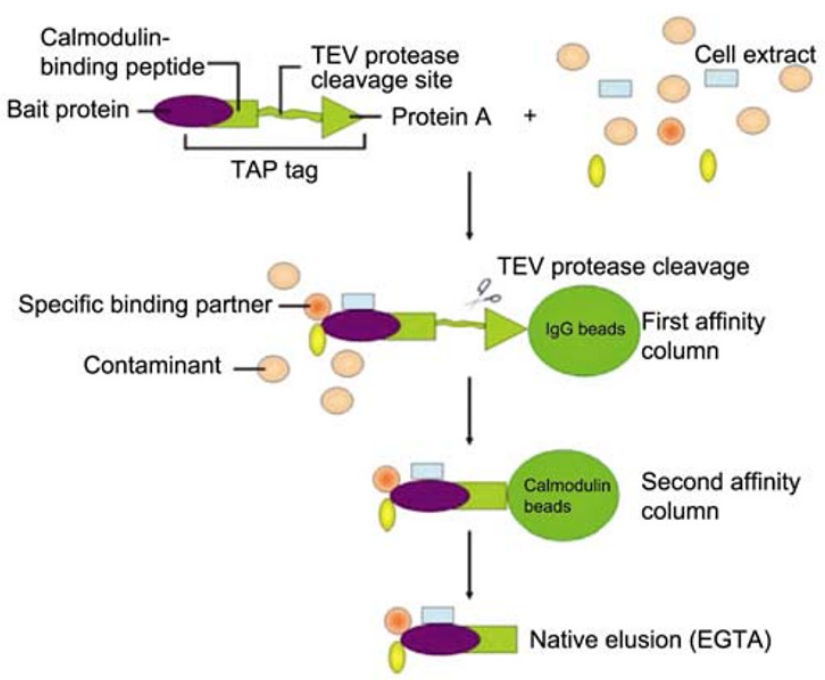

Figure 2 Schematic model of yeast two-hybrid ( $\mathrm{Y} 2 \mathrm{H})$ system and TAP immunopurification methods for the characterization of viral protein interactions. In the $\mathrm{Y} 2 \mathrm{H}$ method, two plasmids including a bait-encoding protein fused to the C-terminus of a transcription factor BD (binding domain) and a prey-encoding protein fused to an AD (activation domain) are constructed. Subsequently, the plasmids are introduced into a suitable yeast strain, and if the two proteins physically interact with one another, the BD and AD are brought together to reconstitute an active transcription factor. This active transcription factor binds to upstream specific activation sequences in the reporter gene promoter to activate expression. By measuring the expression of a reporter gene, the interaction between the proteins can be examined. In the TAP immunoprecipitation method, a fusion tag comprising two sites, one protein A IgG-binding domain and one CBP tag, separated by a spacer containing the specific recognition site for the TEV protease, is expressed in the target cell. The purification steps for interacting proteins are summarized in the right picture. AD, activation domain; BD, DNA-binding domain; CBP, calmodulin-binding peptide; TAP, tandem affinity purification; TEV, tobacco etch virus.

mortalization process. By surveying the differential protein expression profile between pre- and post-immortal cells, this study ultimately identified 20 proteins from 32 spots. Upregulated proteins included proliferating cell nuclear antigen, nucleoside diphosphate kinase-A, and ubiquitin C-terminal hydrolase. Downregulated proteins included ubiquitin ligase $\mathrm{N}$ and stathmin [49]. Coiras et al. [50] examined the impact of the HIV-I tat protein on Jurkat T cells using 2-D DIGE followed by MS analysis. This study noted downregulation of several cytoskeletal proteins, such as actin, $\beta$-tubulin, and annexin II, as well as gelsolin, cofilin, and the Rac/Rho-GDI complex. This expression pattern change could account for the survival of long-term reservoirs of $\mathrm{HIV}$-infected $\mathrm{CD}^{+} \mathrm{T}$ cells responsible for continuous viral production.

\subsection{SILAC (stable isotope labeling by amino acids in cell culture)}

The recently established quantitative proteomic technology, SILAC, has shown great potential in protein expression profiling. SILAC involves a straightforward metabolic labeling method, where the cells are grown in medium containing amino acids with stable nonabundant isotopes (e.g., ${ }^{15} \mathrm{~N},{ }^{13} \mathrm{C}$ and ${ }^{2} \mathrm{D}$ ) and compared with the cells grown in medium containing stable abundant isotopes $\left({ }^{14} \mathrm{~N},{ }^{12} \mathrm{C}\right.$ and $\left.{ }^{1} \mathrm{H}\right)$. Protein abundance is calculated as the ratios of the peak intensity of the fragment ions from the labeled versus the unlabeled peptides, thus constituting an ideal tool for protein expression analysis upon viral infection [51]. Mannova et al. [52] used 2DE followed by mass spectrometry or SILAC combined with one-dimensional electrophoresis separation and mass spectrometry to quantify the modification of the host lipid raft proteome upon HCV infection. While the 2DE and mass spectrometry strategy identified approximately 100 protein spots, the number of individual proteins characterized by the SILAC approach totaled 1036. Many of these proteins were involved in vesicular and protein trafficking and cell signaling. Using small interfering RNAs modulating small GTPases Cdc42 and RhoA, this study noticed an increase in HCV replication. On the other hand, decreased syntaxin 7 expression led to decreased replication of HCV. This proteomics study indicated that protein subcellular relocalization occurs in HCV-containing cells, which can directly influence HCV replication. Recently, Zhang et al. [53] developed a cell line expressing a SARS-CoV subgenomic replicon and used it to screen inhibitors of SARS-CoV replication. The protein profiles of the SARS-CoV replicon cells and parental BHK21 cells were compared by SILAC coupled with mass spectrometry to identify the host proteins essential for SARS-CoV RNA replication. Among the 1081 host proteins quantified, 74 had significantly altered levels of expression. Of these, BCL2-associated athanogene 3 (BAG3), a multifunctional 
molecule proved to participate in cell survival, cellular stress response, proliferation, migration, and apoptosis, was subjected to further functional studies. The results indicated that inhibition of BAG3 expression by RNA interference led to significant suppression of SARS-CoV replication, suggesting a critical role of BAG3 in SARS-CoV replication.

\subsection{Post-experimental labeling methods}

The SILAC approach relies on pre-experimental labeling to analyze the expression level of proteins, and is limited to in vitro investigation. Conversely, chemical isotope labeling targets the proteins or peptides after lysis (post experimental labeling). Theoretically, this approach is applicable to both in vitro and in vivo samples. The amine $-\mathrm{NH}_{2}$, thiol-SH or $-\mathrm{COOH}$ reactive groups of proteins or peptides are isotopically labeled and the samples are mixed equally and analyzed. Widely used methods in this category are isotopecoded affinity tag (ICAT), which targets thiol groups, isobaric tags for relative and absolute quantification (iTRAQ), which targets all the amine groups in the peptides, and ${ }^{18} \mathrm{O}$-labeling, which targets the carboxyl group of peptides during enzymatic hydrolysis [51]. For the ICAT approach, although affinity purification of cysteine-containing peptides significantly reduces the sample complexity, it also prevents the identification and quantitation of peptides containing cysteines and a trypsin-cleavable site. ICAT, 2DE, and multidimensional liquid chromatography coupled to tandem mass spectrometry (2D-LC MS/MS) were used to identify the differential expression profile of proteins upon infection of Atlantic salmon with infectious hematopoietic necrosis virus. The majority of changes in expression levels of proteins, such as natural killer cell enhancement factor (NKEF), procathepsin L, superoxide-producing NADPH oxidase, and interferon-induced viral resistance protein $\mathrm{Mx}$ (IFI-Mx) appeared to be associated with the infectious process [54]. In 2006, Go et al. [55] analyzed the proteome of Drosophila cells infected by flock house virus using the stable isotope labeling method ICAT. This study characterized 1500 cellular proteins from the host, including 150 upregulated and 66 downregulated proteins. Functional classification revealed that these differentially expressed proteins belonged to cellular replication, apoptotic, and metabolic pathways. A more recent study surveyed the global differential protein expression in Vero E6 cells upon SARS-CoV infection. Different proteomic strategies, comprising 2D-GE-ESI-MS/MS using Cy3-Cy5 labeling for detection of bands and ICAT-labeling coupled with 2D-LC-MS/MS, defined the alterations in viral protein expression and host cell protein expression levels. The $2 \mathrm{DE}$ method identified 63 proteins belonging to 48 unique gene products. By contrast, the ICAT approach identified 322 proteins, with 119 upregulated proteins and 48 downregulated proteins, implying a higher sensitivity of ICAT in detecting differentially expressed gene products compared with the traditional 2DE method [56].

Isobaric mass tags for relative and absolute quantitation (iTRAQ) allows simultaneous quantitation of protein extracts from host cells upon virus infection. Li et al. [19] utilized shotgun proteomics involving offline coupling of an LC system with MALDI-TOF/TOF MS/MS as a complementary approach to investigate the WSSV proteome. Forty-five viral proteins were identified, of which 13 were reported for the first time and seven were found to have acetylated $\mathrm{N}$ termini. RT-PCR confirmed the mRNA expression of 13 newly identified viral proteins. The iTRAQ technique was further used to differentiate the envelope proteins and nucleocapsid proteins of WSSV. Twenty-three envelope proteins and six nucleocapsid proteins were successfully identified based on iTRAQ ratios, demonstrating that iTRAQ is an effective approach for high throughput viral protein determination. iTRAQ labeling coupled with LC-MS/MS was also used to investigate the proteomes of a grouper embryonic cell line (GEC) infected with Singapore grouper iridovirus (SGIV) and uninfected GEC. Forty-nine viral proteins were recognized, among which 11 were reported for the first time. Furthermore, 743 host proteins were uncovered and categorized into 218 unique protein groups. Thus, iTRAQ analysis of SGIV infection in GEC provided a novel insight into viral and host gene products at the protein level [57].

Among the various stable isotopic labeling methods, the newly established ${ }^{18} \mathrm{O}$-labeling is the most cost-effective, in that the labeling reagent is heavy water $\left(\mathrm{D}_{2} \mathrm{O}\right)$. However, the major limitation of this approach lies in the fact that the analysis of the low mass difference between peptides, typically 2-4 atomic mass units, requires a highly sensitive MS instrument [58]. In a recent report, ${ }^{18} \mathrm{O}$-labeling was used to study host-virus interactions between HIV-1 and the $\mathrm{HIV}$-infected $\mathrm{CD}^{+}$cell line CEMx174. The cellular proteome of CEMx174 cell line upon HIV-1 infection was assessed using stable isotope labeling, ${ }^{18} \mathrm{O} /{ }^{16} \mathrm{O}$, and accurate mass and time (AMT) tagging. The ${ }^{18} \mathrm{O} /{ }^{16} \mathrm{O}$ ratio was subsequently quantified with a highly sensitive FTICR-MS instrument. 3255 unique host proteins were identified, of which 344 were upregulated and 343 were downregulated. Functional analysis unraveled changes in proteins from various functional categories, including nuclear transport, ubiquitination, cell cycle progression, and the citrate cycle. At least seven proteins (CASP3, DLD, KPNA3, KPNB1, RAN, TPR, and UBE1) were further validated by Western blotting [59].

Taken together, although there have been only a tiny number of large-scale screens of host-virus interactions (HCV, herpesviruses, and West Nile virus), the data seems encouraging. However, the majority of host-virus research has focused on small-scale investigations. The data available can only give us a limited view of the diverse biology of host-virus interactions. More data from a more diverse set of screens and techniques is necessary to achieve a com- 
plete picture of all host-virus interactions.

\section{Conclusion and perspectives}

The rapid advances in MS-based proteomics strategies have facilitated the global analysis of not only virion proteomes, but also the detection and identification of novel virionassociated viral and cellular proteins. The $\mathrm{Y} 2 \mathrm{H}$ system, TAP-tagging method, in vitro pull-down assays, and immunoprecipitation techniques have detected seminal protein-protein interactions between viruses and their corresponding host cells. RNAi screening technology has further characterized the physiological significance of these interactions, which have been corroborated by mutations or other functional experiments. As for protein expression analysis, several isotope-labeling techniques have been able to complement the traditional 2DE coupled with mass spectrometry strategy to identify global alterations in cell protein levels during viral infection. However, the functions of most of those identified virion-associated proteins, as well as alterations in cellular proteomes or novel protein interactions, have yet to be characterized. Moreover, protein interaction screen results, using either the $\mathrm{Y} 2 \mathrm{H}$ system or protein complex purification and mass spectrometry, are expected to increase exponentially in the future. This will require matching advances in computational biology techniques to model, visualize, and analyze the detailed interaction maps occurring during the viral infection processes. These viral proteomics technologies hold much promise for improving our understanding of virus-host interactions. Such an understanding will ultimately aid the diagnosis of specific infectious diseases, as well as help in the design of novel antiviral drugs and vaccines for various virus-human interactions and for targeted treatments.

This work was supported by the National Project on Major Infectious Diseases Prevention (Grant No. 2008ZX10002-009), and the National Basic Research Program of China (Grant No. 2011CB910703).

1 Karen L M, Lori F. Viral proteomics. Microbiol Mol Biol Rev, 2007, 71: 398-411

2 Yuan K, Lei Y L, Huang C. Application of chemistry-based functional proteomics to screening for novel drug targets. Comb Chem High Throughput Screen, 2010, 13: 414-421

3 Liu R, Bai S J, Li Z J, et al. Mechanism of cancer cell adaptation to metabolic stress: proteomics identification of a novel thyroid hormone-mediated gastric carcinogenic signalling pathway. Mol Cell Proteomics, 2009, 8: 70-85

4 Miklos G L, Maleszka R. Protein functions and biological contexts. Proteomics, 2001: 169-178

5 Nair K S, Asmann Y W, Short K R, et al. Proteomic research: potential opportunities for clinical and physiological investigators. Am J Physiol Endocrinol Metab, 2004, 286: 863-874

6 Fields B N, Howley P M. Fundamental virology. In: Principles of Virus Structure, 3rd ed. Philadelphia: Lippincott, Williams \& Willkins, 1996. 59-100

7 Kalkkinen N, Soderlund H, Kaariainen L. Analysis of semliki-for- est-virus structural proteins to illustrate polyprotein processing of alpha viruses. Eur J Biochem, 1980, 108: 31-37

8 Chelius D, Shieh C H, Lehmberg E, et al. Analysis of the adenovirus type 5 proteome by liquid chromatography and tandem mass spectrometry methods. J Proteome Res, 2002, 1: 501-513

9 Davison A J, Davison M D. Identification of structural proteins of channel catfish virus by mass spectrometry. Virology, 1995, 206: 1035-1043

10 Resch W G, Moore R J, Lipton M S, et al. Protein composition of the vaccinia virus mature virion. Virology, 2007, 358: 233-247

11 Chung C S, Ho M Y, Huang C Y, et al. Vaccinia virus proteome: identification of proteins in vaccinia virus intracellular mature virion particles. J Virol, 2006, 80: 2127-2140

12 Yoder J D, Gagnier C R, Vemulapalli S, et al. Pox proteomics: mass spectrometry analysis and identification of Vaccinia virion proteins. Virol J, 2006, 3: 10-26

13 Baldick C J, Shenk T. Proteins associated with purified human cytomegalovirus particles. J Virol, 1996, 70: 6097-6105

14 Kattenhorn L M, Wagner M, Lomsadze A, et al. Identification of proteins associated with murine cytomegalovirus virions. J Virol, 2004, 78: 11187-11197

15 Johannsen E, Chase M R, Weicksel S, et al. Proteins of purified Epstein-Barr virus. Proc Natl Acad Sci USA, 2004, 101: 16286-16291

16 Zhu F X, Wu L J, Yuan Y. Virion proteins of Kaposi's sarcomaassociated herpesvirus. J Virol, 2005, 79: 800-811

17 O'Connor C M, Kedes D H. Mass spectrometric analyses of purified Rhesus monkey rhadinovirus reveal 33 virion-associated proteins. J Virol, 2006, 80: 1574-1583

18 Huang C H, Lin Q S, Xu X, et al. Proteomic analysis of shrimp white spot syndrome viral proteins and characterization of a novel envelope protein VP466. Mol Cell Proteomics, 2002, 1: 223-231

19 Li Z, Chen J, Wu J L, et al. Shotgun identification of the structural proteome of shrimp white spot syndrome virus and iTRAQ differentiation of envelope and nucleocapsid subproteomes. Mol Cell Proteomics, 2007, 6: 1609-1620

20 Zeng R, Jiang X S, Zhou H, et al. Proteomic analysis of SARS associated coronavirus using two-dimensional liquid chromatography mass spectrometry and one-dimensional sodium dodecyl sulfate-polyacrylamide gel electrophoresis followed by mass spectroemtric analysis. J Proteome Res, 2004, 3: 549-555

21 Saphire A C, Bark S J. Proteomic analysis of human immunodeficiency virus using liquid chromatography/tandem mass spectrometry effectively distinguishes specific incorporated host proteins. J Proteome Res, 2006, 5: 530-538

22 Chertova E, Coren L V, Roser J D, et al. Proteomic and biochemical analysis of purified human immunodeficiency virus type 1 produced from infected monocyte-derived macrophages. J Virol, 2006, 80: 9039-9052

23 Bortz E, Whitelegge J P, Jia Q M, et al. Identification of proteins associated with murine gammaherpesvirus 68 virions. J Virol, 2003, 77 : 13425-13432

24 Varnum S M, Monroe M E, Smith P, et al. Identification of proteins in human cytomegalovirus (HCMV) particles: the HCMV proteome. J Virol, 2004, 78: 10960-10966

25 Rozen R, Li Y, Yuan Y. Virion-wide protein interactions of Kaposi's sarcoma-associated herpesvirus. J Virol, 2008, 82: 4742-4750

26 Bartel P L, Roecklein J A, SenGupta D, et al. Host and viral proteins in the virion of Kaposi's sarcoma-associated herpesvirus. J Virol, 2005, 79: 4952-4964

27 Vliet K V, Zhang L L, Villa N Y, et al. Poxvirus proteomics and virus-host protein interactions. Microbiol Mol Biol Rev, 2009, 73: 730-749

28 Oliver K B, Russel J D, Anthony L C. New insights into viral structure and virus-cell interactions through proteomics. Expert Rev Proteomics, 2005, 2: 577-588

29 Bortz E, Whitelegge J P, Jia Q, et al. Identification of proteins associated with murine gammaherpesvirus 68 virions. J Virol, 2003, 77: 13425-13432

30 Ying W T, Zhang Y J, Peng W M, et al. Proteomic analysis on struc- 
tural proteins of severe acute respiratory syndrome coronavirus. Proteomics, 2004, 4: 492-504

31 Savalia D, Westblade L F, Goel M, et al. Genomic and proteomic Analysis of phiEco32, a novel Esacherichia coli bacteriophage. J Mol Biol, 2008, 377: 774-789

32 Robert M D, Martin N L, Kropinski A M. The genome and proteome of coliphage T1. Virology, 2004, 318: 245-266

33 Naryshkina T, Liu J, Florens L, et al. Thermus thermophilus bacteriophage phiYS40 genome and proteomic characterization of virions. J Mol Biol, 2006, 364: 667-677

34 Beijerinck M J. Concerning a contagium vivum fluidum as cause of the spot disease of tobacco leaves. Verhandelingen der Koninkyke akademie Wettenschapppen te Amsterdam, 1898, 65: 3-21

35 Fields S, Song O. A novel genetic system to detect protein-protein interactions. Nature, 1989, 340: 245-246

36 Fields S. Interactive learning: Lessons from two hybrids over two decades. Proteomics, 2009, 9: 5209-5213

37 Mendez-Rios J, Uetz P. Global approaches to study protein-protein interactions among viruses and hosts. Future Microbiol, 2010, 5: 289-301

38 Bartel P L, SenGupta D, Fields S. A protein linkage map of Escherichia coli bacteriophage T7. Nat Genet, 1996, 12: 72-77

39 McCraith S, Moss B, Fields S. Genome-wide analysis of vaccinia virus protein-protein interactions. Proc Natl Acad Sci USA, 2000, 97 : 4879-4884

40 Dziembowski A, Ventura A P, Rutz B, et al. Proteomic analysis identifies a new complex required for nuclear pre-mRNA retention and splicing. EMBO J, 2004, 23: 4847-4856

41 Jorba N, Torreira E, Gastaminza P, et al. Analysis of the interaction of influenza virus polymerase complex with human cell factors. Proteomics, 2008, 8: 2077-2088

42 Mayer D, Martinez-Sobrido L, Ghanem A, et al. Identification of cellular interaction partners of the influenza virus ribonucleoprotein complex and polymerase complex using proteomic-based approaches. J Proteome Res, 2007, 6: 672-682

43 Molina-Jiménez F, Murata M, Martín-Vílchez S, et al. Expression of pituitary tumor-transforming gene 1 (PTTG1)/securin in hepatitis B virus (HBV)-associated liver diseases: evidence for an HBV X protein-mediated inhibition of PTTG1 ubiquitination and degradation. Hepatol, 2010, 51: 777-787

44 Cristea I M, Carroll J N, Rout M P, et al. Tracking and elucidating alphavirus-host protein interactions. J Biol Chem, 2006, 281: 30269-30278

45 Hirsch A J. The use of RNAi-based screens to identify host proteins involved in viral replication. Future Microbiol, 2010, 5: 303-311
46 Brass A L, Dykxhoor D M, Benita Y, et al. Identification of host proteins required for HIV infection through a functional genomics screen. Science, 2008, 319: 921-926

47 Konig R, Zhou Y Y, Elleder D, et al. Global analysis of host-pathogen interactions that regulate early-stage HIV-1 replication. Cell, 2008, 135: 49-60

48 Tong A P, Gou L T, Lau Q C, et al. Proteomic profiling identifies aberrant epigenetic modifications induced by hepatitis B virus $\mathrm{X}$ protein. J Proteome Res, 2009, 8: 1037-1046

49 Toda T, Sugimoto M, Omori A, et al. Proteomic analysis of Epstein-Barr virus-transformed human B-lymphoblastiod cell lines before and after immortalization. Electrophoresis, 2000, 21: 1814-1822

50 Coiras M, Camafeita E, Urena T, et al. Modifications in the human T cell proteome induced by intracellular HIV-1 Tat protein expression. Proteomics, 2006, 6: S63-S73

51 Chen X, Yu Y B, Xue Y, et al. Amino acid-coded tagging approaches in quantitative proteomics. Expert Rev Proteomics, 2007, 4: 25-37

52 Mannova P, Wang H, Deng B, et al. Modification of host lipid raft proteome upon hepatitis $\mathrm{C}$ virus replication. Mol Cell Proteomics, 2006, 5: 2319-2325

53 Zhang L, Zhang X E, Lin F S, et al. Quantitative proteomics analysis reveals BAG3 as a potential target to suppress severe acute respiratory syndrome coronavirus replication. J Virol, 2010, 84: 6050-6059

54 Booy A T, Ohlund L B, Hardie D B, et al. Application of isotope coded affinity tag (ICAT) analysis for the identification of differentially expressed proteins following infection of atlantic salmon (Salmo salar) with infectious hematopoietic necrosis virus (IHNV) or Renibacterium salmoninarum (BKD). J Proteome Res, 2005, 4: 325-334

55 Go E P, Wikoff W R, Shen Z, et al. Mass spectrometry reveals specific and global molecular transformations during viral infection. $\mathrm{J}$ Proteome Res, 2006, 5: 2405-2416

56 Jiang X S, Tang L Y, Dai J, et al. Quantitative analysis of severe acute respiratory syndrome (SARS)-associated coronavirus-infected cells using proteomic approach. Mol Cell Proteomics, 2005, 4: 902-913

57 Chen L M, Lin Q S, Lim T K, et al. iTRAQ analysis of Singapore grouper iridovirus infection in a grouper embryonic cell line. J General Virol, 2008, 89: 2869-2876

58 Diamond D L, Jacobs J M, Paeper B, et al. Proteomic profiling of human liver biopsies: hepatitis $\mathrm{C}$ virus-induced fibrosis and mitochondrial dysfunction. Hepatol, 2007, 46: 649-657

59 Chan E Y, Qian W J, Diamond D L, et al. Quantitative analysis of HIV-1 infected CD4 ${ }^{+}$cell proteome: dysregulated cell cycle progression and nuclear transport coincide with robust virus production. $\mathrm{J}$ Virol, 2007, 81: 7571-7583

Open Access This article is distributed under the terms of the Creative Commons Attribution License which permits any use, distribution, and reproduction in any medium, provided the original author(s) and source are credited. 